\title{
CORRECTION
}

\section{Correction to: A simple approach for the preoperative assessment of sacral morphology for percutaneous SI screw fixation}

\section{Michael Goetzen ${ }^{1} \cdot$ Vinzent Kevin Ortner ${ }^{1} \cdot$ Richard A. Lindtner $^{1} \cdot$ Rene Schmid $^{1} \cdot$ Michael Blauth $^{1}$.} Dietmar Krappinger ${ }^{1}$

Published online: 17 April 2018

๑) Springer-Verlag GmbH Germany, part of Springer Nature 2018

\section{Correction to: \\ Arch Orthop Trauma Surg (2016) 136:1251-1257 \\ https://doi.org/10.1007/s00402-016-2528-3}

In the original article, co-author's given name has been published incorrectly.

The correct given name should be Vinzent Kevin.

The original article can be found online at https://doi.org/10.1007/ s00402-016-2528-3.

Dietmar Krappinger

dietmar.krappinger@i-med.ac.at

Michael Goetzen

goetzen.michael@gmail.com

Vinzent Kevin Ortner

kevin.ortner@student.i-med.ac.at

Richard A. Lindtner

richard.lindtner@tirol-kliniken.at

Rene Schmid

rene.schmid@tirol-kliniken.at

Michael Blauth

michael.blauth@i-med.ac.at

1 Department of Trauma Surgery, Medical University

of Innsbruck, Anichstraße 35, 6020 Innsbruck, Austria 